\title{
Immunofluorescence study of actin, acrosin, dynein, tubulin and hyaluronidase and their impact on in-vitro fertilization
}

\author{
Alfred Senn', Marc Germond and Pierre De Grandi \\ Fertility Unit, Department of Obstetrics and Gynaecology, Centre \\ Hospitalier Universitaire Vaudois, CH-1011 Lausanne, Switzerland \\ ${ }^{1}$ To whom correspondence should be addressed
}

Using polyclonal antibodies, the distribution of actin, acrosin, dynein, tubulin and hyaluronidase has been examined by indirect immunofluorescence in sperm preparations from fertile donors and in-vitro fertilization (IVF) patients. After recording sperm parameters in native semen, spermatozoa were washed free of seminal plasma using either the swimup or the Percoll filtration technique. Prior to insemination, aliquots of the washed sperm suspensions were prepared for antibody staining. Spermatozoa from fertile donors were analysed in order to establish the specific fluorescence patterns of each antibody and the threshold scores of normality. Immunofluorescence scores obtained from IVF patients were then analysed with respect to IVF outcome. For each tested protein, the number of normal samples were significantly lower in the group which did not fertilize and fertilization rates were significantly reduced when any of the tested proteins were scored as pathological. Normal fluorescence scores were correlated with morphology, motility, velocity and to a lesser extent with sperm concentration in native semen. On the basis of receiver-operating characteristic curves, likelihood ratios and Cohen's kappa values, the presence of acrosin and tubulin yields the most useful information on sperm functional and structural status and on its fertilizing ability.

Key words: acrosome/immunofluorescence/in-vitro fertilization/ polyclonal antibodies

\section{Introduction}

In cases of male or unexplained infertility, seminal analyses and functional tests have a limited ability to predict the outcome of in-vitro fertilization (IVF) (Edwards et al., 1984; Oehninger and Alexander, 1991). Successful fertilization relies on several associated sperm characteristics, such as concentration, motility and morphology (Tesarik and Testart, 1989; Acosta et al., 1989), capacitation, zona binding and acrosome reaction (Wolf, 1989), membrane fusion, DNA decondensation and nuclear fusion. Any test or combination of tests to predict the outcome of fertilization should provide information on the ability of spermatozoa to undergo each of these various steps.

Several sperm proteins are involved in fertilization at the structural or functional level. Actin has been found in all mammalian species studied so far (Ochs and Wolf, 1985; Flaherty et al., 1988). Actin, which is present in the fibrous sheath of the tail (Flaherty et al., 1988), was also found in the subacrosomal space during spermatogenesis and in the equatorial region and postacrosomal sheath of developed spermatozoa, where this protein is thought to play various possible roles in membrane-related events during capacitation and fertilization (Vogl, 1989). Acrosin is an essential component of the acrosomal matrix (de Vries et al., 1985) and is involved in two critical steps of fertilization, i.e. homologous zona binding and zona penetration (Topfer Petersen and Henschen, 1987; Tesarik et al., 1988). Total activity of both the active zymogen form and inactive proacrosin were shown to correlate with zygote formation (Kennedy et al., 1989). Furthermore, an indirect immunofluorescence study using a monoclonal anti-acrosin antibody, has shown a positive correlation between normal head morphology and the presence of an intact fluorescing acrosome (Francavilla et al., 1988). Antibody against the inactive proacrosin has also been obtained and used successfully to detect progressive loss of the acrosome during capacitation (Gallo et al., 1991).

Dynein and tubulin are part of the axoneme organization and are both involved in microtubule sliding and tail movement. Hyaluronidase is present in the spermatozoa of many mammalian species (Mack et al., 1983; Singer $e t$ al., 1983; Hirayama $e t$ al., 1989) and is secreted by the acrosome in order to facilitate penetration by spermatozoa through the egg vestments (Triana et al., 1980; Lewin et al., 1982).

A large amount of literature has focused on identification of these proteins in mammalian spermatozoa (Gallo et al., 1986; Florke Gerloff et al., 1987; Kaufmann et al., 1987; Francavilla et al., 1988; Baccetti et al., 1989), but correlation of indirect immunofluorescence results with fertilization is still lacking. Using polyclonal antibodies, the distribution of actin, acrosin, dynein, tubulin and hyaluronidase has been examined by indirect immunofluorescence in sperm preparations of IVF patients and fertile donors. The present study was aimed at establishing the correlations between IVF and the detection by immunofluorescence of these various proteins.

\section{Materials and methods}

\section{Sperm preparation and fertilization}

Semen samples were obtained from normozoospermic donors $(n=12)$ or from patients enrolled in our IVF programme $(n=132)$. After complete liquefaction of the ejaculates at $37^{\circ} \mathrm{C}$ for 30-45 min, sperm count and motility were measured with 
an automated semen analyser (Hamilton-Thom Motility Analyser, Hamilton-Thorn, 30A Cherry Hill Drive, Danvers, Massachusetts 01923, USA). Morphology was assessed according to the usual standards on Papanicolaou stained smears (World Health Organization, 1987). Spermatozoa were washed free of seminal plasma by self-migration of the motile cells from $1 \mathrm{ml}$ native semen into a $2 \mathrm{ml}$ of overlay of human tubal fluid (HTF) medium (Quinn et al., 1985), containing $0.5 \%$ bovine serum albumin (BSA, Sigma, A 4161, USA) (Benadiva et al., 1989). In pathological samples, spermatozoa were sedimented by centrifugation $(600 \mathrm{~g}, 20 \mathrm{~min})$ through a step gradient (Forster et al., 1983), prepared by carefully layering $1 \mathrm{ml}$ fractions of $90 \%, 80 \%$ and $50 \%$ Percoll (Pharmacia, Sweden) in isotonic Ham's-F10 culture medium (Gibco, 074-01200, Scotland, UK). The recovered spermatozoa were then washed twice by centrifugation $(300 \mathrm{~g}, 10 \mathrm{~min}$ ) in $2 \mathrm{ml}$ HTF. The final pellet was resuspended in $0.4-1.0 \mathrm{ml} \mathrm{HTF}$ and sperm count, motility and morphology were reassessed.

Oocytes were obtained by ultrasound-guided follicle puncture from patients undergoing ovarian stimulation with a gonadotrophin-releasing hormone analogue (Decapeptyl, Ferring, Zürich) and human menopausal gonadotrophin (HMG) protocols (Germond et al., 1990). Oocytes were pre-incubated in $0.9 \mathrm{ml}$ HTF for 4-6 h, inseminated with $0.5-2.0 \times 10^{5}$ spermatozoa in $100 \mu \mathrm{l}$ and further incubated at $36.9^{\circ} \mathrm{C}\left(5 \% \mathrm{O}_{2}, 5 \% \mathrm{CO}_{2}\right.$, $90 \% \mathrm{~N}_{2}$ ) for $16 \mathrm{~h}$. Eggs were then separated from the remaining corona cells by aspiration through a finely drawn Pasteur pipette and checked for the presence of pronuclei under phase contrast illumination $(\times 400)$. Zygotes were transferred into $1 \mathrm{ml}$ of Menezo B2 culture medium (BioMérieux, France) and further incubated under the same conditions for $24 \mathrm{~h}$. Cleaved embryos were replaced as previously described (De Grandi and Germond, 1987).

No selection criteria were applied to either male or female partners.

\section{Immunofluorescence}

Molecular probes for indirect immunofluorescence were kindly provided by Serono Diagnostics, Trading Division (SpermaScreen kit, Biodata, Rome, Italy). For each sperm sample, five smears were prepared with $5 \mu \mathrm{l}$ aliquots of the washed sperm suspensions, which were adjusted to a concentration range of $1-60 \times$ $10^{6} / \mathrm{ml}$. Sample preparation for indirect immunofluorescence was done according to Baccetti et al. (1989). Air-dried (30-60 min) smears were fixed with cold methanol $\left(-20^{\circ} \mathrm{C}, 15 \mathrm{~min}\right)$ and acetone $\left(-20^{\circ} \mathrm{C}, 5 \mathrm{~min}\right)$. For organizational purposes, staining was performed on batches on 10 samples. Slides were thus either kept frozen at $-20^{\circ} \mathrm{C}$ until use or processed immediately for indirect immunofluorescence staining. No differences between fresh or cryopreserved samples could be detected when samples were stored at $-20^{\circ} \mathrm{C}$ for $\leq 4$ weeks.

Spermatozoa were rehydrated $(2 \times 5 \mathrm{~min})$ in phosphatebuffered saline (PBS), treated for 15 min with $50 \mu$ normal goat serum (5\% in PBS containing 1\% BSA) to prevent non-specific binding, and reacted overnight at $4^{\circ} \mathrm{C}$ in a humidified chamber with the various rabbit polyclonal antiboodies (pAb), diluted (1:50) in PBS containing $0.1 \%$ BSA. Slides were then carefully washed with PBS $(3 \times 10 \mathrm{~min})$ and incubated for $1 \mathrm{~h}$ at room temperature with $50 \mu$ l of fluorescein isothiocyanate (FITC)-labelled goat antirabbit IgG antibody, diluted in PBS (1:50). After three washes in PBS ( $3 \times 10 \mathrm{~min})$, slides were mounted with $32 \times 24 \mathrm{~mm}$ coverslips in PBS:glycerol (10:90) containing 2.5-5\% propylgallate (Sigma, P3130, USA) to prevent fading, and examined using an epifluorescence microscope (Olympus BH2S, FITC illumination, Y455 filter) at $\times 400-\times 1000$ magnification. Unlabelled cells were briefly visualized under phase-contrast illumination. For each tested pAb, 100 spermatozoa were counted per slide and fluorescence scored as normal or pathological, according to the patterns described in Figure 1 and Table I.

\section{Acrosin activity determination}

The procedure of Kennedy et al. (1989), based on hydrolysis of $N$ - $\alpha$-benzoyl-DL-arginine $p$-nitroanilide hydrochloride (BAPNA, Sigma, USA) at pH 8.0, was used with the following modifications. An aliquot $(0.3 \mathrm{ml})$ of the washed sperm suspension in HTF was washed by centrifugation $(300 \mathrm{~g}, 10 \mathrm{~min})$ in $2 \mathrm{ml}$ PBS. The pellet was resuspended in exactly $0.3 \mathrm{ml}$ PBS, frozen at $-20^{\circ} \mathrm{C}$ and stored for $\leq 2$ weeks. Thawed samples were divided into three $100 \mu \mathrm{l}$ aliquots in conical centrifuge tubes. The first aliquot received $100 \mu$ l benzamidine hydrochloride (500 mM, Sigma, USA), the two others were duplicate assays. Incubation in the presence of $1 \mathrm{ml}$ of the substrate-detergent mixture was performed at $22-25^{\circ} \mathrm{C}$ and stopped after $3 \mathrm{~h}$ with $100 \mu$ l benzamidine. Cell debris was pelleted by centrifugation $(800 \mathrm{~g}, 20 \mathrm{~min})$ and the supernatant used for colourimetric assay $(410 \mathrm{~nm})$. Not all IVF samples could be analysed, as in some cases the whole sperm suspension had to be used for IVF.

\section{Statistical analysis}

Unpaired Student's $t$-test, chi-square analysis or Fisher's test were performed wherever appropriate. Receiver-operating characteristic curves were derived from IVF results using various cutoff values (Richardson et al., 1985), for which the likelihood ratios and Cohen's kappa values (Collins, 1989) were also calculated.

\section{Results}

\section{Qualitative evaluation of fluorescence patterns in spermatozoa from fertile donors}

Typical fluorescence patterns observed with the five pAb tested are illustrated in Figure 1. Observations gained from the fertile donors are summarized in Table I.

Actin was clearly seen along the equatorial segment and the head-tail junction (centriolar region), while the acrosomal cap and posterior portion of the head fluoresced to a variable degree from sample to sample. Fluorescence of the midpiece increased with the amount of cytoplasm still present; diffuse fluorescence was seen along the principal piece of the tail. The following patterns were considered as pathological: no fluorescence along the equatorial segment, fluorescence distributed uniformly over the whole head or limited to its periphery or no fluorescence along the principal piece. 
a
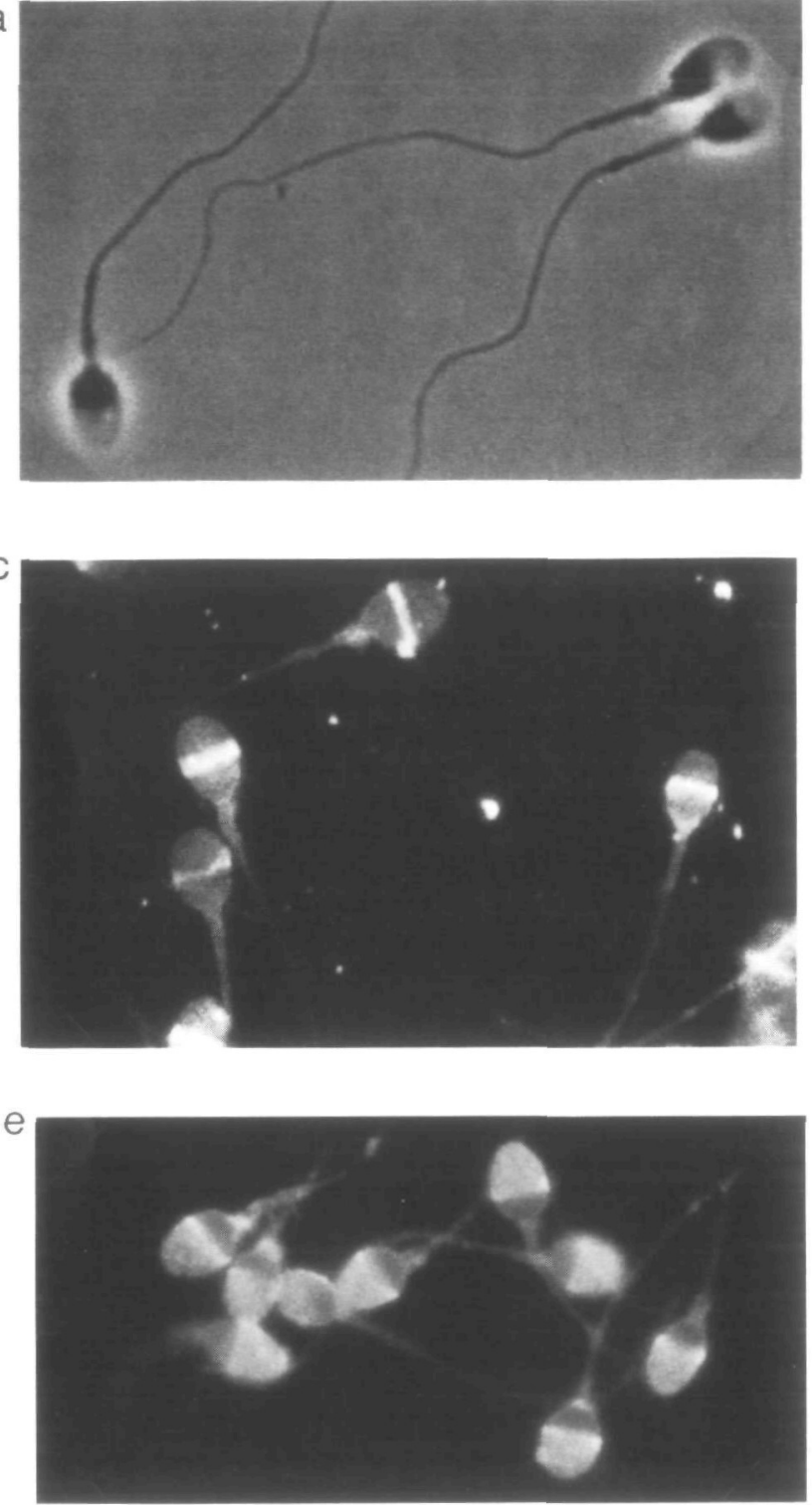

b

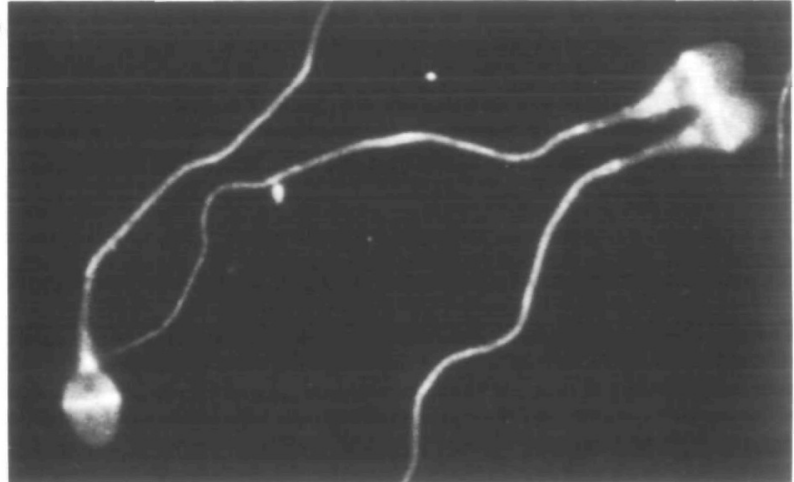

d
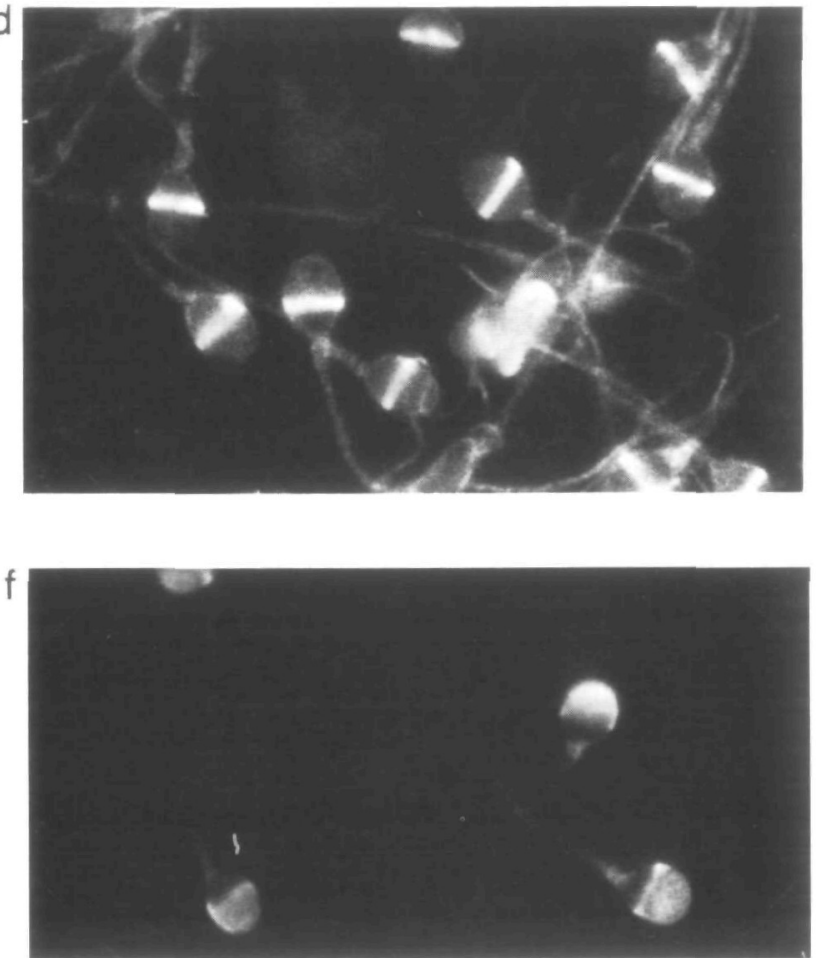

Fig. 1. Phase contrast (a) and indirect immunofluorescence of spermatozoa obtained from fertile donors using polyclonal antibodies against actin (b), dynein (c), tubulin (d), acrosin (e) and hyaluronidase (f).

With the anti-acrosin pAb, the acrosomal cap was clearly visible and fluoresced at variable intensities: staining could appear homogeneously distributed over the acrosome or restricted to small patches. Acrosin was considered absent when the acrosomal region was not fluorescent or, as for actin, when fluorescence was distributed over the whole head or restricted to its periphery.

Dynein was seen along the equatorial segment and in many cases over the acrosome. The tail was usually faintly stained. Tubulin had fluorescence patterns similar to dynein, except for the acrosome which was usually not stained, and for the tail which was brightly fluorescent. Hyaluronidase was confined to the acrosomal cap.

Cells which did not exhibit fluorescence at locations marked positive on Table I were counted as pathological. The indirect immunofluorescence score was then determined by the percentage of cells exhibiting the expected fluorescence patterns. Mean immunofluorescence scores \pm SD were established using
Table I. Localization of fluorescence in the spermatozoa of fertile donors using various polyclonal antibodies ( $\mathrm{pAb}$ )

\begin{tabular}{lllll}
\hline pAb against & Head & & Midpiece & Tail \\
\cline { 2 - 3 } & Acrosome & Equatorial segment & & \\
\hline Actin & V & P & V & P \\
Acrosin & P & P & V & V \\
Dynein & V & P & V & P \\
Tubulin & V & P & V & P \\
Hyaluronidase & P & N & N & N \\
\hline
\end{tabular}

$P, N, V$ : positive, negative, weak or varable staining.

-Some cells may show no fluorescence as a result of spontaneous

completion of the acrosome reaction or structural lack of the acrosomal cap.

samples obtained from a fertile population (Table II). For all proteins, the mean indirect immunofluorescence scores were at least $90 \%$. 
Table II. Normal fluorescence scores ( $(\%)$ determined in sperm samples from fertile donors $(n=18)$

\begin{tabular}{llllll}
\hline & Actin & Acrosin & Dynein & Tubulun & Hyaluronidase \\
\hline Mean \pm SD & $94.2 \pm 9.9$ & $90.2 \pm 14.0$ & $92.7 \pm 11.4$ & $93.9 \pm 9.7$ & $97.0 \pm 6.8$ \\
Mean -2SD & 70 & 60 & 70 & 70 & 80
\end{tabular}

$\mathrm{SD}=$ standard deviation.

Mean-2SD = lower normality threshold rounded to the nearest 10 of the flworescence scores used to discriminate between normal and pathological samples.

Tabłe III. Percentage of normal samples and mean indirect immunofluorescence scores for five sperm proteins, observed in IVF cycles with or without fertilization

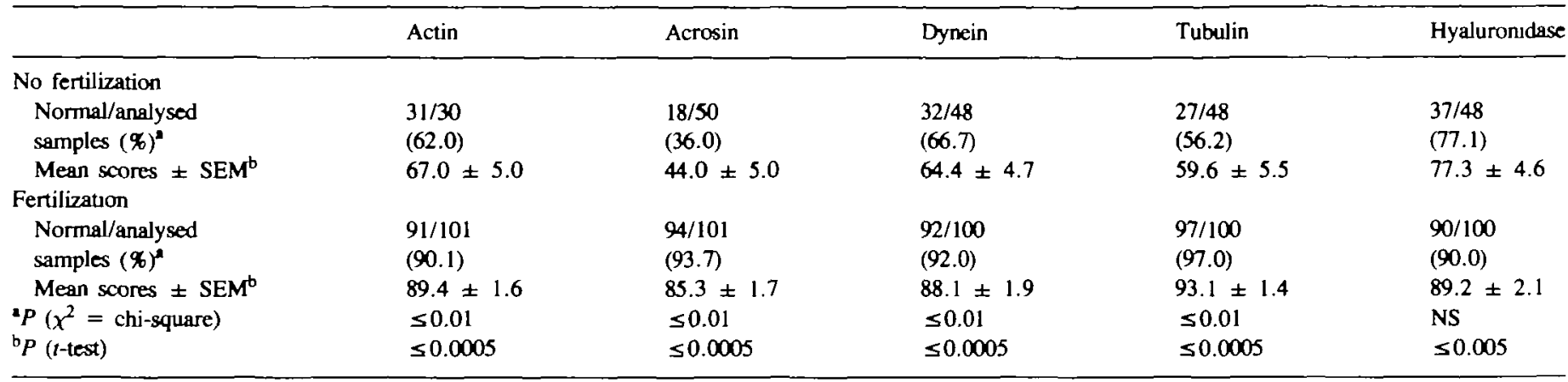

Table IV. Fertilization rates achieved by samples sorted according to the WHO cnterba or indirect immunofluorescence scores as normal or pathological

\begin{tabular}{|c|c|c|c|c|c|c|}
\hline & $\begin{array}{l}\text { WHO } \\
\text { criteria }\end{array}$ & Actin & Acrosin & Dynein & Tubulin & Hyaluronidase \\
\hline \multicolumn{7}{|l|}{ Normal: } \\
\hline Zygotes/oocytes" & $50.4 \pm 3.6$ & $45.5 \pm 3.4$ & $49.3 \pm 3.4$ & $43.8 \pm 3.3$ & $46.8 \pm 3.3$ & $42.4 \pm 3.4$ \\
\hline$(\%)$ & $(83.0)$ & $(75.4)$ & (83.9) & $(75.8)$ & $(79.8)$ & $(72.4)$ \\
\hline \multicolumn{7}{|l|}{ Pathological: } \\
\hline Zygotes/oocytes" & $22.9 \pm 4.5$ & $14.6 \pm 5.0$ & $11.5 \pm 4.1$ & $20.7 \pm 7.2$ & $4.8 \pm 4.1$ & $25.5 \pm 7.4$ \\
\hline$P(t-\operatorname{test})^{2}$ & $\leq 0.0005$ & $\leq 0.0005$ & $\leq 0.0005$ & $\leq 0.005$ & $\leq 0.0005$ & $\leq 0.05$ \\
\hline
\end{tabular}

${ }^{a}$ Mean (in $\%$ ) \pm SEM.

${ }^{\mathrm{b}} P \leq 0.05 ;{ }^{c} P \leq 0.01$.

Six donors were tested twice at 1-2 month intervals and yielded highly reproducible fluorescence patterns. The interassay coefficient of variation was $<10 \%$ for each tested protein. No significant differences in staining patterns were observed between the swim-up and Percoll methods of sperm preparation.

\section{Correlation of indirect immunofluorescence results with IVF}

In order to evaluate the clinical impact of the immunofluorescence study, IVF samples were defined as either normal or pathological using a lower threshold of normality, which was arbitrarily defined by the mean $-2 S D$ of the immunofluorescence scores measured on donor samples (Table II). The number of normal samples were counted in IVF patients who did or did not achieve fertilization (Table III). In the group with fertilization $\sim 90 \%$ of the patients were scored as normal. The number of normal scores was significantly lower in the group without fertilization for all sperm proteins except hyaluronidase $(77.1 \%)$. The lowest percentages of normal samples were found for acrosin (36.0\%)

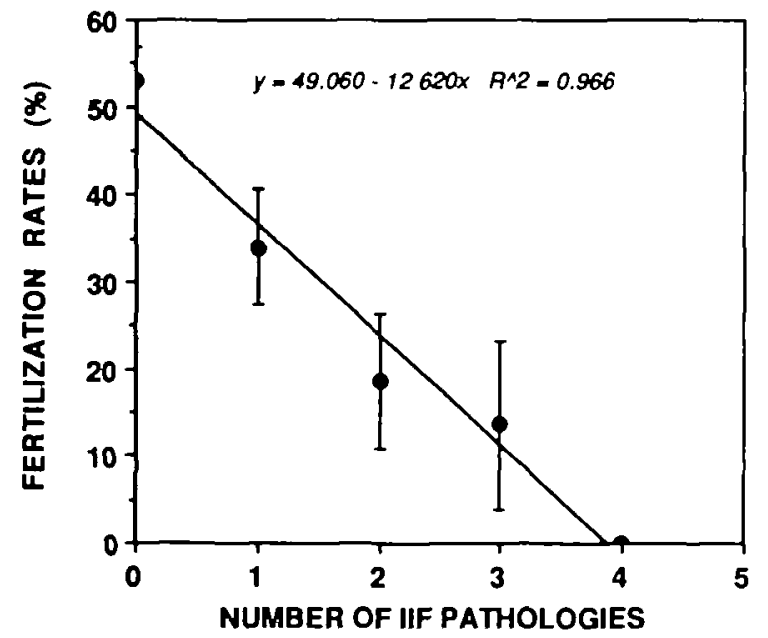

Fig. 2. Correlation between number of indirect immunofluorescence (IIF) pathologies/sample and fertilization rates in sperm samples from IVF patients. 
and tubulin (56.2\%). Mean immunofluorescence scores, calculated in the two groups, were significantly lower in the absence of fertilization for all proteins and below the threshold values gained from fertile donors.

Data were also analysed in terms of fertilization rates achieved among samples sorted according to the WHO criteria or immunofluorescence scores (Table IV). Fertilization rates, expressed as mean zygotes/oocytes or transfers/cycle were significantly lower when any of the tested proteins had abnormal immunofluorescence scores. These results highlight the possibility that each protein studied could be involved at some point with fertilization; abnormal samples, however, often exhibited more than one pathological feature simultaneously. In order to study this aspect, IVF samples were sorted by increasing numbers of abnormalities. As shown in Figure 2, the corresponding fertilization rates dropped sharply as spermatozoa were affected by an increasing number of abnormalities.

\section{Correlation of immunofluorescence results with other sperm parameters}

Indirect immunofluorescence scores and seminal parameters (sperm count, mobility and morphology) known to be correlated with fertilization were submitted to regression analysis. Correlation coefficients $(r)$ and statistical significance are summarized in Table V. Immunofluorescence scores of hyaluronidase showed little or no correlation with the various parameters. In contrast, the scores of the other proteins were positively correlated, especially with normal morphology and rapid progressive motility, determined as the number of cells ( $\%$ ) exhibiting a velocity $\geq 25 \mu / \mathrm{s}$. The presence of acrosin and hyaluronidase did not correlate with native sperm count, nor was the presence of dynein associated with sperm velocity. The tubulin score was significantly dependent on all native semen parameters and especially on sperm velocity $(r=0.48-0.50 ; P \leq 0.005)$.

\section{Correlation of acrosin immunofluorescence scores with enzymatic activity}

An indirect check of the specificity of the pAb for acrosin was achieved by evaluating indirect immunofluorescence in parallel to the enzymatic activity of acrosin from lysed sperm cells preincubated at $\mathrm{pH}$ 8.0. Figure 3 summarizes the data obtained from 96 samples from the 153 IVF attempts, for which the number of cells recovered after washing was high enough to allow measurement of acrosin activity on the same cell suspension as that used for IVF. Immunofluorescence scores for acrosin were significantly correlated ( $P \leq 0.005$ ) with the corresponding acrosin activities, suggesting that the $\mathrm{pAb}$ recognized acrosin. No correlation was found, however, between the intensity of fluorescence (recorded subjectively as weak, medium or high) and the enzymatic activity (data not shown), indicating that the $\mathrm{pAb}$ might not have recognized both the active and inactive forms of acrosin. In Figure 3, a distinction was made between cycles with or without fertilization. In the restricted population studied, no fertilization occurred when the immunofluorescence score was $<60 \%$ (vertical bar), which corresponded to the lower normal threshold of acrosin (Table II).
Table V. Correlation coefficients between normal immunofluorescence scores and seminal parameters in sperm samples from IVF patients $(n=153)$

\begin{tabular}{|c|c|c|c|c|c|}
\hline $\begin{array}{l}\text { Seminal } \\
\text { parameters }\end{array}$ & Actin & Acrosin & Dynein & Tubulin & Hyaluronidase \\
\hline $\begin{array}{l}\text { Native sperm } \\
\text { count } \\
\left(10^{6} / \mathrm{ml}\right)\end{array}$ & $0.35^{* *}$ & 0.16 & $0.39 * *$ & $0.34^{* *}$ & 0.017 \\
\hline $\begin{array}{l}\text { Rapid } \\
\text { progressive } \\
\text { motility }\left(\boldsymbol{X}^{2}\right)^{*}\end{array}$ & $0.55 * * *$ & $0.40^{* * *}$ & $0.39^{* *}$ & $0.45^{* *}$ & 0.18 \\
\hline $\begin{array}{l}\text { Curvilinear } \\
\text { velocity } \\
(\mu \mathrm{m} / \mathrm{s})\end{array}$ & $0.35^{* *}$ & $0.29 * *$ & $0.22 *$ & $0.48 * * *$ & $0.25^{*}$ \\
\hline $\begin{array}{l}\text { Progressive } \\
\text { velocity } \\
(\mu \mathrm{m} / \mathrm{s})\end{array}$ & $0.27^{*}$ & $0.19^{*}$ & 0.17 & $0.50^{* * *}$ & 0.17 \\
\hline $\begin{array}{l}\text { Normal } \\
\text { morphology } \\
(\%)\end{array}$ & $0.60^{* * *}$ & $0.51^{* * *}$ & $0.62^{* * *}$ & $0.51^{* * *}$ & $0.25^{*}$ \\
\hline
\end{tabular}

${ }^{*} P \leq 0.05,{ }^{* * P} \leq 0.005, * * * P \leq 0.0005$.

Rapid progressive motility is the number of cells (\%) exhibiting a velocity $\geq 25 \mu / \mathrm{s}$.

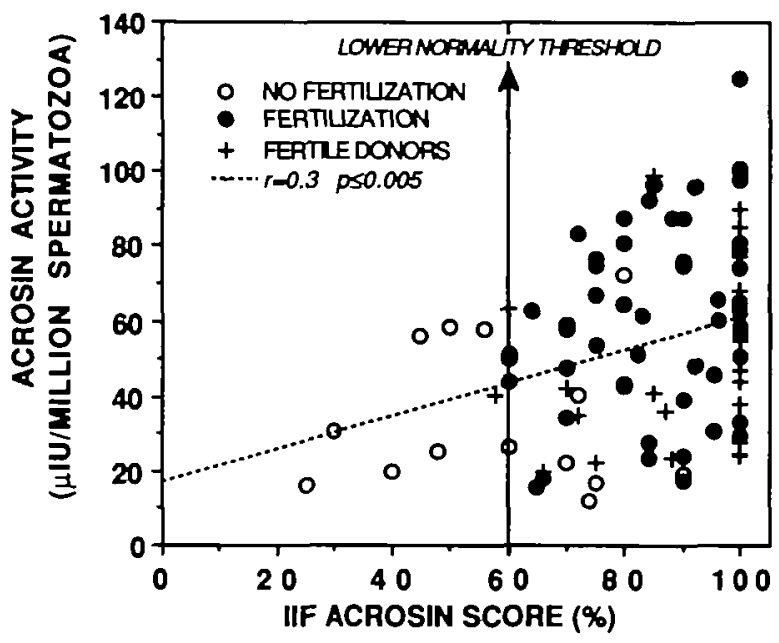

Fig. 3. Correlation between indirect immunofluorescence antiacrosin scores and acrosin activities in 96 samples of lysed sperm cells from IVF attempts.

\section{Comparison of immunofluorescence scores and sperm parameters as predictors of IVF outcome}

In order to obtain a threshold value yielding the highest clinical relevance, various threshold values were tried and the corresponding sensitivity, specificity, likelihood ratios and Cohen's kappa statistic were calculated for the five tested proteins as well as for the classical semen parameters. Results obtained for acrosin (immunofluorescence scores and activity) and normal morphology are shown in Table VI. For acrosin immunofluorescence scores, the best threshold value was found to be $\sim 60 \%$, confirming the threshold gained from the fertile donor population (Table II). Sensitivity and specificity for the $60 \%$ acrosin immunofluorescence score was 0.92 and 0.63 respectively. Likelihood ratios were in the fair range, while the corresponding kappa value 
Table VI. Comparison of morphology and acrosin indirect immunofluorescence (IIF) status and activity as predictors of fertilization in vitro in sperm samples from IVF patients

\begin{tabular}{|c|c|c|c|c|c|c|}
\hline Methods & Threshold & Sensituvity & Specificity & LR+ & LR - & Kappa \\
\hline \multirow{6}{*}{$\begin{array}{l}\text { Acrosin } \\
\text { indirect } \\
\text { immunofluorescence }\end{array}$} & $20^{n}$ & 0.99 & 0.31 & 1.43 & 0.03 & 0.624 \\
\hline & 40 & 0.99 & 0.41 & 1.67 & 0.02 & 0.669 \\
\hline & 50 & 0.99 & 0.55 & 2.21 & 0.02 & 0.736 \\
\hline & 60 & 0.92 & 0.63 & 2.51 & 0.12 & 0.706 \\
\hline & 80 & 0.74 & 0.80 & 3.60 & 0.33 & 0.606 \\
\hline & 100 & 0.39 & 0.90 & 3.84 & 0.68 & 0.385 \\
\hline \multirow{6}{*}{$\begin{array}{l}\text { Acrosin } \\
\text { activity }\end{array}$} & $15^{b}$ & 1.00 & 0.07 & 1.07 & 0.00 & 0.659 \\
\hline & 20 & 0.94 & 0.27 & 1.29 & 0.21 & 0.659 \\
\hline & 30 & 0.87 & 0.53 & 1.86 & 0.25 & 0.659 \\
\hline & 40 & 0.79 & 0.60 & 1.98 & 0.35 & 0.600 \\
\hline & 50 & 0.70 & 0.73 & 2.61 & 0.41 & 0.545 \\
\hline & 70 & 0.36 & 0.93 & 5.38 & 0.69 & 0.320 \\
\hline \multirow[t]{4}{*}{ Morphology } & $20^{c}$ & 1.00 & 0.07 & 1.07 & 0.00 & 0.542 \\
\hline & 40 & 0.97 & 0.34 & 1.47 & 0.09 & 0.624 \\
\hline & 50 & 0.90 & 0.52 & 1.89 & 0.18 & 0.643 \\
\hline & 80 & 0.28 & 0.95 & 6.09 & 0.76 & 0.327 \\
\hline
\end{tabular}

\%o cells exhibiting normal fluorescence patterns.

$\mathrm{b}_{\mu \mathrm{IU} / \text { million spermatozoa. }}$

c\% cells with normal morphology according to WHO.

LR +: likelihood of fertilization given an abnormal test $(2-5=$ fair agreement).

LR-: likelihood of fertilization given a normal test $(0.1-0.2=\operatorname{good}$ agreement).

Kappa: Cohen's kappa $(0.40-0.75=$ good agreement $)$.

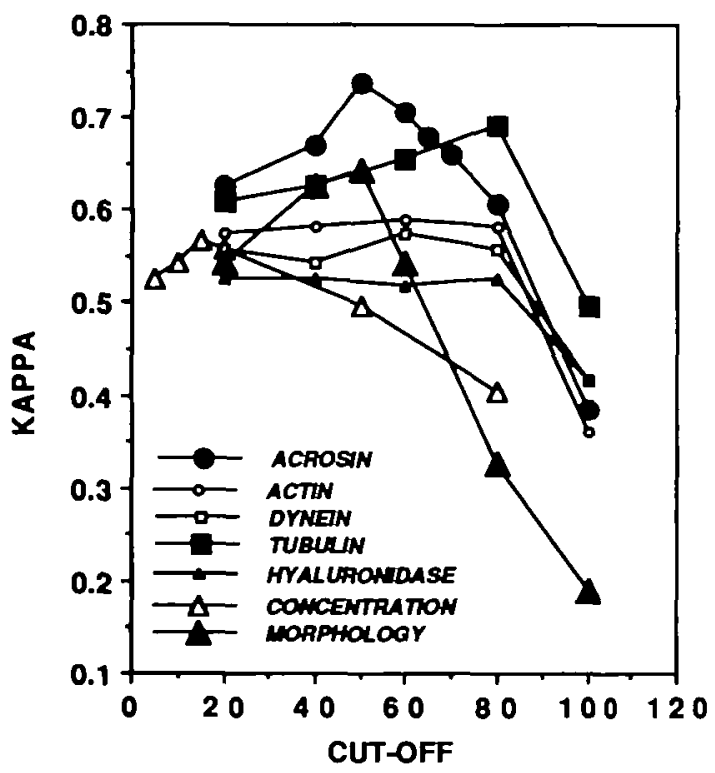

Fig. 4. Cohen's kappa values plotted against various thresholds of normality for the five tested proteins as well as for morphology and sperm counts in native semen.

was good (0.706). Normal morphology yielded similar sensitivity and specificity at the 50\% threshold; the likelihood of fertilization given an abnormal or normal test $(\mathrm{LR}+$ and $\mathrm{LR}-$ ) were slightly less significant and the kappa value diminished in comparison to acrosin immunofluorescence.

Kappa values were plotted against various cut off thresholds of normality for the five tested proteins as well as the morphology

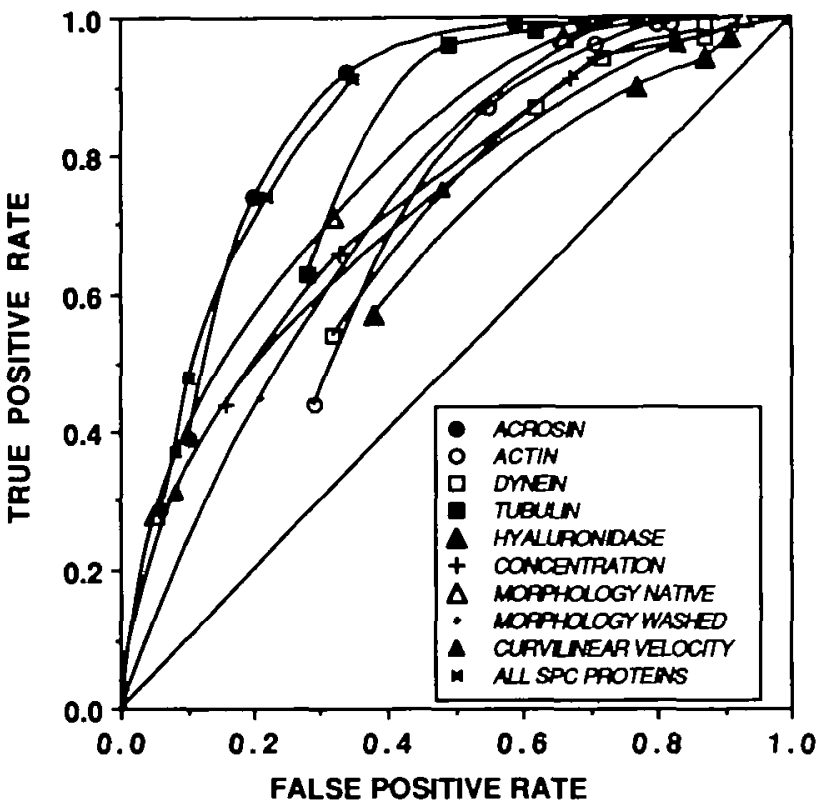

Flg. 5. Receiver-operating characteristic curves for various discriminating parameters used to predict IVF outcome. Diagonal represents a putative test with no clinical significance yielding the same incidence of true and false positives for all thresholds.

Tabłe VII. Percentage of normal indirect immunofluorescence samples sorted according to WHO criteria and ferulization

\begin{tabular}{|c|c|c|c|c|c|c|c|}
\hline \multirow[t]{2}{*}{ Fertilization } & \multirow{2}{*}{$\begin{array}{l}\text { WHO } \\
\text { criteria }\end{array}$} & \multirow[t]{2}{*}{$n$} & \multicolumn{5}{|c|}{ Normal samples (\%) with respect to: } \\
\hline & & & Actin & Acrosin & Dynein & Tubulin & Hyaluronidase \\
\hline \multirow[t]{2}{*}{ Failure } & Normal & 16 & 87.3 & $56.3^{4}$ & 87.5 & 87.5 & 93.8 \\
\hline & Abnormal & 33 & $57.6^{d}$ & $27.3^{b}$ & 57.6 & $39.4^{\mathrm{c}}$ & 66.7 \\
\hline \multirow[t]{2}{*}{ Success } & Normal & 78 & 99.4 & $94.9^{n}$ & 96.2 & 97.4 & 92.3 \\
\hline & Abnormal & 24 & $91.7^{d}$ & $83.3^{b}$ & 79.2 & $91.7^{c}$ & 83.3 \\
\hline
\end{tabular}

a.b.c $P \leq 0.001,{ }^{d} P \leq 0.02$.

and sperm counts in native semen (Figure 4). Acrosin yielded the highest kappa values with a maximum at a threshold of $50 \%$. The curve obtained for morphology had a similar shape, but with lower values over the entire range. In contrast, no clear maximum was found for the other proteins with the possible exception of tubulin. Sperm concentration had a maximum $\sim 15-20 \times 10^{6}$ spermatozoa/ml, which corresponded to the value recommended by the WHO.

The data were submitted to receiver-operating characteristic (ROC) curve analysis (Figure 5). ROC curves were plotted for various seminal parameters and for the various immunofluorescence scores, by varying the cut-off points of normality. The diagonal represented a putative test with no clinical significance, i.e. a test yielding the same incidence of true and false positives for all thresholds. The more accurate a test was in predicting fertilization, the more the corresponding ROC curve departed from the diagonal. The results presented in Figure 5 show that acrosin immunofluorescence was clearly superior to conventional semen analysis in predicting fertilization.

Finally, sperm samples were sorted according to the WHO 
criteria of normality (i.e. $\geq 20 \times 10^{6} / \mathrm{ml}, \geq 25 \%$ rapid motility, $\geq 50 \%$ normal morphology) and to IVF outcome (Table VII). Among the 57 samples considered as abnormal on the basis of the WHO criteria, 24 samples (42\%) were able to fertilize and $33(58 \%)$ were not. In the same groups, the percentage of samples classified as normal by indirect immunofluorescence was significantly higher when fertilization was successful compared to when it failed, in particular for actin (91.7 versus $57.6 \% ; P \leq 0.02$ ), acrosin (83.3 versus $27.3 \% ; P \leq$ $0.001)$ and tubulin $(91.7 \%$ versus $39.4 \% ; P \leq 0.001)$. In the samples classified as normal by the WHO criteria, the percentage of normal samples with respect to immunofluorescence was similar whether fertilization occurred or not, except in the case of acrosin, which dropped from $94.9 \%$ normal samples in the presence of fertilization to $56.3 \%$ when it failed ( $P \leq 0.001$ ). These results suggest that indirect immunofluorescence screening of sperm samples before the first IVF attempt is particularly useful, whatever the semen quality, as it allows the identification of those abnormal WHO samples which are likely to fertilize; and among normal WHO samples, it would explain, in $\sim 50 \%$ of the cases, why fertilization failed, i.e. due to an abnormal level of acrosin.

\section{Discussion}

Diagnostic tests aimed at predicting the outcome of IVF rely on either structural or functional assessment of the spermatozoa. With the currently available tests which can be performed prior to IVF, the ability to obtain a clear distinction between subfertile and infertile sperm samples is, however, still controversial (Oehninger and Alexander, 1991), and some authors consider that IVF is the only reliable test (Liu et al., 1988; Acosta et al., 1989). Morphology estimated according to strict criteria has so far been the best parameter to predict fertilization outcome (Kruger et al., 1987). Immunofluorescence offers an alternative method to describe some particular properties of the sperm cell, since the sperm proteins which are suspected to play a critical role in fertilization may be visualized and their proper localization in spermatozoa assessed.

In this study, immunofluorescence scores of all proteins, except hyaluronidase, were highly correlated with normal morphology (Table V); consequently, it is not surprising that the immunofluorescence scores were higher in samples which achieved fertilization than in those which failed (Table III). Hyaluronidase, located in the acrosome, was poorly correlated with morphology (Table V) and was not clearly related to IVF outcome (Table III). This finding corroborates the absence of correlation between hyaluronidase activity and the acrosomal pathologies reported by Singer et al. (1983), which could indicate that hyaluronidase is rarely deficient, and confirms the observation that cumulus cells, once incubated with spermatozoa in vitro, are always dispersed regardless of the sperm quality.

The best correlations between immunofluorescence scores and fertilization rates were observed for acrosin and tubulin (Tables IV, VI, VII, Figures 4 and 5). ROC curves, which allow various diagnostic tests to be compared, showed that immunofluorescence scores for acrosin and tubulin could more effectively predict fertilization outcome than more conventional parameters, such as sperm counts, normal morphology or curvilinear velocity (Figure 5). In order to determine the best threshold values for each tested protein, we submitted our data to critical evaluation by calculating for various thresholds of normality, the corresponding sensitivity, specificity, likelihood ratios and Cohen's kappa (Table VI, Figure 4). This analysis confirmed the presence of a clear maximum in the kappa values for acrosin, tubulin, normal morphology and to a lesser extent sperm count (Figure 4). The highest kappa values were obtained for acrosin (0.736) and tubulin $(0.690)$ at thresholds of $50 \%$ and $80 \%$ respectively, which indicates that these tests could be superior to the more conventional parameters, such as morphology (0.643 at 50\%) or sperm count $\left(0.555\right.$ at $\left.15 \times 10^{6} / \mathrm{ml}\right)$. The other antibodies tested (e.g. anti-actin, -dynein or -hyaluronidase) did not produce any significant improvement over the conventional diagnostic methods (Figures 4 and 5).

Immunofluorescence distributions observed with the polyclonal antibodies are comparable to those described in the literature for actin (Flaherty et al., 1988), acrosin (Francavilla et al., 1988; Baccetti et al., 1989), dynein (Baccetti et al., 1988) and hyaluronidase (de Vries et al., 1985; Baccetti et al., 1989). In the case of tubulin, clear staining of the equatorial segment, besides that of the principal piece of the tail, was observed. This puzzling observation was also made by Virtanen et al. (1984) and Gallo et al. (1986), who used monoclonal antibodies to $\alpha$ tubulin.

In this first study, we did not test in great detail whether the various antibodies were specific to the respective proteins, as the various fluorescence patterns detected were consistent with detailed descriptions in the literature (Baccetti et al., 1988). However, an attempt to assess the specificity of the acrosin antibody was performed by comparing the immunofluorescence scores with the acrosin activity determined on the sperm samples used for IVF (Figure 3). A significant correlation was found, although the acrosin activities were subject to large fluctuations from sample to sample (Kennedy et al., 1989; Liu and Baker, 1990). Unlike Kennedy et al. (1989), we were unable to find any clear threshold value below which fertilization would fail (Table VI). This could be due to the washing of spermatozoa, which selects the cells that are most likely to fertilize and consequently tends to reduce the differences in acrosin activity between the samples that will or will not fertilize. As cell count is used as the denominator, it is noteworthy that the calculated activity may be subject to technical errors, especially for low sperm densities $\left(<5 \times 10^{6}\right.$ spermatozoa/ml).

Results of this study agree with those of Kruger et al. (1988) and Liu and Baker (1990), who found no relationship between acrosin activity and fertilization in vitro. There is no doubt that this enzyme plays a critical role during zona binding and zona penetration (Tesarik et al., 1988) but its action is dependent on structural and biochemical events which take place during capacitation and the acrosome reaction. The advantage of the indirect immunofluorescence approach is that acrosin can be detected in its proper location, i.e. the acrosome, which could explain the discrepancy observed between the good diagnostic value of the acrosin immunofluorescence score and the poor diagnostic value of acrosin activity. Acrosin immunofluorescence scores exhibit the widest variation range among fertile donors 
and consequently have the lowest threshold of normality (Table II). This reflects the known heterogeneity of acrosomal status in a given ejaculate, as some cells spontaneously complete the acrosomal reaction or lose their acrosomal contents during washing.

In conclusion, indirect immunofluorescence scores for acrosin and tubulin are significantly correlated with the fertilizing ability of spermatozoa and may be of invaluable help in determining the probability of fertilization prior to IVF, especially in cases of poor sperm morphology and idiopathic infertility.

\section{Acknowledgements}

In part supported by Serono Diagnostics, Trading Division, Coinsins, Switzerland.

\section{References}

Acosta,A.A., Oehninger,S., Morshedi,M., Swanson,R.J., Scott,R. and Irianni,F. (1989) Assisted reproduction in the diagnosis and treatment of the male factor. Obstet. Gynecol. Surv., 44, 1-18.

Baccetti,B., Burrini,A.G., Collodel,G., Magnano,A.R., Piomboni,P. and Renieri,T. (1988) Immunocytochemistry and sperm pathology. J. Submicrosc. Pathol., 20, 209-224.

Baccetti,B., Burrini,A.G., Collodel,G., Piomboni,P. and Renieri,T. (1989) Localization of acrosomal enzymes in Arthropoda, Echinodermata and Vertebrata. J. Submicrosc. Cytol. Pathol., 21, $385-389$.

Benadiva,C.A., Kuczynski Brown,B., Maguire,T.G., Mastoianni,L.,Jr and Flickinger,G.L. (1989) Bovine serum albumin (BSA) can replace patient serum as a protein source in an in-vitro fertilization (IVF) program. J. In Vitro Fertil. Embryo Transfer, 6, 164-167.

Collins,J.A. (1989) Male infertility: the interpretation of the diagnostic assessment. In Mishell,D.R., Paulsen,C.A. and Lobo,R.A. (eds), The Year Book of Infertility. Year Book Medical Publishers, Chicago, London, Boca Raton, pp. 45-74.

De Grandi,P. and Germond,M. (1987) Technique du transfert d'embryons. In Revaz,C. (ed.), Fécondation In Vitro et Transfert d'Embryons. Médecine et hygiène, Genève, pp. 83-88.

Edwards,R.G., Fishel,S.B., Cohen,J., Fehilly,C.B., Purdy,J.M., Slater,J.M., Steptoe,P.C. and Webster,J.M. (1984) Factors influencing the success of in-vitro fertilization for alleviating human infertility. J. In Vitro Fertil. Embryo Transfer, 1, 3-23.

Flaherty,S.P., Winfrey,V.P. and Olson,G.E. (1988) Localization of actin in human, bull, rabbit, and hamster sperm by immunoelectron microscopy. Anat. Rec., 221, 599-610.

Florke Gerloff,S., Topfer Petersen,E., Schill,W.B. and Engel,W. (1987) Evolution and development of the outer acrosomal membrane (OAM) and evidence that acrosin-inhibitors are proteins of the OAM. Andrologia, 19, 121-133.

Forster,M.S., Smith,W.D., Lee,W.I., Berger,R.E. and Karp,L.E. (1983) Selection of human spermatozoa according to their relative motility and their interaction with zona-free hamster eggs. Fertil. Steril., 40, 655-660.

Francavilla,S., Bruno,B., Poccia,G., Francavilla,F., Properzi,G. and Santucci,R. (1988) Fluorescence microscopic detection of acrosin in different morphologic types of human spermatozoa. Andrologia, 20, $344-350$.

Gallo,J.M., Escalier,D., Schrevel,J. and David,G. (1986) Differential distribution of tubulin epitopes in human spermatozoa. Eur. J. Cell Biol., 40, $111-116$.

Gallo,J.M., Escalier,D., Grellier,P., Precigout,E., Albert,M., David,G. and Schrevel,J. (1991) Characterization of a monoclonal antibody to human proacrosin and its use in acrosomal status evaluation. $J$. Histochem. Cytochem., 39, 273-282.

Germond,M., Senn,A., Reymond,O. and De Grandi,P. (1990) Why lose an in-vitro fertilization cycle when stimulation fails. Fertil. Steril., 53, 936-938.

Hirayama,T., Hasegawa,T. and Hiroi,M. (1989) The measurement of hyaluronidase activity in human spermatozoa by substrate slice assay and its clinical application. Fertil. Steril., 51, 330-334.

Kaufmann,M., Schonwald,D., Mansouri,A. and Gunther,E. (1987) Immunological approach to characterize proacrosin and various acrosin forms in boar and man by monoclonal antibodies. Gamete Res. , 18, $349-361$.

Kennedy,W.P., Kaminski,J.M., Van der,V.H.H., Jeyendran,R.S., Reid,D.S., Blackwell,J., Bielfeld,P. and Zaneveld,L.J. (1989) A simple, clinical assay to evaluate the acrosin activity of human spermatozoa. J. Androl., 10, 221-231.

Kruger,T.F., Ackermann,S.B., Simmons,K.F., Swanson,R.J., Matta,J.F., Veeck,L.L., Morshedi,M. and Brugo,S. (1987) A quick, reliable staining technique of evaluating sperm morphology with predictive value for human in vitro fertilization. Arch. Androl., 18, $275-277$

Kruger,T.F., Haque,D., Acosta,A.A., Pleban,P., Swanson,R.J. Simmons,K.F., Matta,J.F., Morshedi,M. and Oehninger,S. (1988) Correlation between sperm morphology, acrosin, and fertilization in an IVF program. Arch. Androl., 20, 237-241.

Lewin,L.M., Nevo,Z., Gabsu,A. and Weissenberg, R. (1982) The role of sperm-bound hyaluronidase in the dispersal of the cumulus oophorus surrounding rat ova. Int. J. Androl., 5, 37-44.

Liu,D.Y. and Baker,H.W. (1990) Relationships between human sperm acrosin, acrosomes, morphology and fertilization in vitro. Hum. Reprod., 5, 298-303.

Liu,D.Y., Du Plessis,Y.P., Nayudu,P.L., Johnston,W.I.H. and Baker,H.W.G. (1988) The use of in vitro fertilization to evaluate putative tests of human sperm function. Fertil. Steril., 49, 272-277.

Mack,S., Bhattacharyya,A.K., Joyce,C., van der Ven,H. and Zaneveld,L.J. (1983) Acrosomal enzymes of human spermatozoa before and after in vitro capacitation. Biol. Reprod., 28, 1032-1042.

Ochs,D. and Wolf,D.P. (1985) Actin in ejaculated human sperm cells. Biol. Reprod., 33, 1223-1226.

Oehninger,S. and Alexander,N.J. (1991) Male infertility: the focus shifts to sperm manipulation. Curr. Opin. Obstet. Gynecol., 3, 182-190.

Quinn,P., Kerin,J.F. and Warnes,G.M. (1985) Improved pregnancy rate of human in vitro fertilization with the use of a medium based on the composition of human tubal fluid. Fertil. Steril., 44, 493-498.

Richardson,D.K., Schwartz,J.S., Weinbaum,P.J. and Gabbe,S.G. (1985) Diagnostic tests in obstetrics: a method for improved evaluation. Am. J. Obstet. Gynecol., 152, 613-618.

Singer,R., Sagiv,M., Allalouf,D., Levinsky,H., Barnet,M., Landau,B. and Servadio,C. (1983) Lack of correlation between acrosomal pathologies of human spermatozoa and hyaluronidase activity in sperm. Int. J. Fertil., 28, 119-120.

Tesarik,J. and Testart,J. (1989) Human sperm-egg interactions and their disorders: implications in the management of infertility. Hum. Reprod., 4, 729-741.

Tesarik,J., Drahorad,J. and Peknicova,J. (1988) Subcellular immunochemical localization of acrosin in human spermatozoa during the acrosome reaction and zona pellucida penetration. Fertil. Steril., $\mathbf{5 0}$, $133-141$

Topfer-Petersen,E. and Henschen,A. (1987) Acrosin shows zona and fucose binding, novel properties for a serine proteinase. FEBS Lett., 226, 38-42.

Triana,L.R., Babcock,D.F., Lorton,S.P., First,N.L. and Lardy,H.A. (1980) Release of acrosomal hyaluronidase follows increased membrane permeability to calcium in the presumptive capacitation sequence for spermatazoa of the bovine and other mammalian species. Biol. Reprod., 23, 47-59. 
Virtanen,I., Lehto,P., Kallkoji,M. and Blose,S.H. (1984) Differential localization of $\alpha$ - and $\beta$-uubulin in human sperm cells. J. Cell Biol., 99, 41.

Vogl,A.W. (1989) Distribution and function of organized concentrations of actin filaments of mammalian spermatogenic cells and Sertoli cells. Int. Rev. Cytol., 119, 1-56.

Wolf,D.P. (1989) Acrosomal status quantitation in human sperm. Am. J. Reprad. Immunol., 20, 106-113.

World Health Organization (1987) WHO Laboratory Manual for the Examination of Human Semen and Semen-Cervical Mucus Interaction, 2nd edn. Cambridge University Press, Cambridge, pp. $1-67$.

de Vries,J.W., Willemsen,R. and Geuze,H.J. (1985) Immunocytochemical localization of acrosin and hyaluronidase in epididymal and ejaculated porcine spermatozoa. Eur. J. Cell Biol, , 37, 81-88.

Received on December 11, 1991; accepted on March 26, 1992 